Check for updates

Cite this: RSC Adv., 2021, 11, 12361

Received 4th February 2021

Accepted 9th March 2021

DOI: $10.1039 / \mathrm{d} 1 \mathrm{ra00939g}$

rsc.li/rsc-advances

\section{A Turn-ON fluorometric biosensor based on ssDNA immobilized with a metal phenolic nanomaterial for the sequential detection of $\mathrm{Pb}(\mathrm{II})$ and epirubicin cancer drug $\dagger$}

\begin{abstract}
A. Arunjegan, ${ }^{a}$ P. Rajaji, ${ }^{a}$ S. Sivanesan (D) ${ }^{b}$ and P. Panneerselvam (DD *a
In this paper, we propose a fluorescent biosensor for the sequential detection of $\mathrm{Pb}^{2+}$ ions and the cancer drug epirubicin (Epn) using the interactions between label-free guanine-rich ssDNA (LFGr-ssDNA), acridine orange (AO), and a metal-phenolic nanomaterial (i.e., nano-monoclinic copper-tannic acid (NMc-CUTA)). An exploration of the sensing mechanism shows that LFGr-ssDNA and AO strongly adsorb on NMc-CUTA through $\pi-\pi$ stacking and electrostatic interactions, and this results in the fluorescence quenching of $A O$. In order to sense the target $\mathrm{Pb}^{2+}$, initially, LFGr-ssDNA specifically binds with $\mathrm{Pb}^{2+}$ ions to form a G4 complex $\left(\mathrm{G}-\mathrm{Pb}^{2+}-\mathrm{G}\right.$ base pair), which was released from the surface of NMc-CuTA with strong AO fluorescence enhancement (Turn-ON). The subsequent addition of a biothiol, like cysteine (Cys), to the G4 complex decreases the fluorescence, as the $\mathrm{Pb}^{2+}$ ions released from the G4 complex have a higher interaction affinity with the sulfur atoms of Cys; this further induces the unwinding of the G4 complex to form LFGr-ssDNA. Finally, Epn was added to this, which intercalates with LFGr-ssDNA to form a G4 complex via G-Epn-G, resulting in fluorescence recovery (Turn-ON). Accordingly, the Turn-ON fluorescent probe had subsequent limits of detection of 1.5 and $5.6 \mathrm{nM}$ for $\mathrm{Pb}^{2+}$ and Epn, respectively. Hence, the reported NMc-CUTA-based sensing platform has potential applications for the detection of $\mathrm{Pb}^{2+}$ and Epn in real samples with good sensitivity and selectivity.
\end{abstract}

\section{Introduction}

The detection of toxic heavy metal ions based on sensitivity and selectivity criteria has been recognized as an issue for decades worldwide. $\mathrm{Pb}^{2+}$ ions are specifically the most toxic heavy metal, causing dangerous health disorders in adults and infants. Even low concentrations of $\mathrm{Pb}^{2+}$ consumption have adverse effects, such as hypertension and cardiovascular, reproductive, nervous system, and developmental disorders. The US Environment Protection Agency (EPA) has set the safe limit of $\mathrm{Pb}^{2+}$ in drinking water as $72 \mathrm{nM}^{\mathbf{1 - 3}}$ Epirubicin (Epn) is an anticancer chemotherapeutic drug that exerts cytotoxic effects via inhibiting DNA synthesis and DNA replication. Thus, it finds applications in treating various cancer disorders, primarily breast, ovarian, gastric, and lung cancer, but its application is limited as it has serious side effects, like cardiotoxicity, bone marrow suppression, hair loss, low blood counts, and vomiting, when

${ }^{a}$ Department of Chemistry, SRM Institute of Science and Technology, Kattankulathur, Tamil Nadu, 603 203, India. E-mail: panneerp1@srmist.edu.in; panneerchem82@ gmail.com; Tel: +919688538842

${ }^{b}$ Department of Applied Science and Technology, A. C Technology, Anna University, Chennai, Tamil Nadu, 600 025, India

$\dagger$ Electronic supplementary information (ESI) available. See DOI: 10.1039/d1ra00939g its dosage is high ${ }^{4,5}$. Usually, $\mathrm{Pb}^{2+}$ and Epn cannot be identified based on color, taste, or odour. Therefore, it is very essential for researchers to develop sensitive and rapid detection techniques to measure even trace amounts of $\mathrm{Pb}^{2+}$ and $\mathrm{Epn}$ in biological samples.

Several analytical methods, including atomic absorption spectroscopy, high-performance liquid chromatography (HPLC), electrochemical sensors, cold vapor atomic absorption spectroscopy, and inductively coupled plasma mass spectrometry, have been used for $\mathrm{Pb}^{2+}$ and Epn drug analysis in the laboratory. ${ }^{6-11}$ These described techniques often rely on costly instruments, are time-consuming and operationally tedious, and involve sample pretreatment processes; therefore, it is essential to establish appropriate methodology to overcome these disadvantages. The use of fluorescent biosensors is one of the best analytical tools compared with other techniques, as it involves very high intrinsic sensitivity, rapid analysis times, and simplicity. Currently, many researchers are focusing on the design of fluorescence-based biosensors using label- and labelfree DNA, which binds to specific analytes with unique recognition. ${ }^{\mathbf{1 2 - 1 4}}$ Based on this, large numbers of nanomaterials have been synthesized for the detection of toxic metal ions and biomolecules. 
Upconverting nanoparticles (UCNPs), silicon nanocomposites (SiNCs), graphene-like 2D materials, graphene oxide (GO), transition metal dichalcogenides (TMD), graphitic carbon nitride, and transition metal oxides (TMOs) ${ }^{15-21}$ have attracted great attention as transduction elements and supporting substrates in a wide variety of biosensing technologies. 2D materials can provide an extremely high density of active surface sites over a large surface area, making them ideal for biochemical sensing. Although these nanomaterials have been successfully used for the sensitive and specific fluorescence detection of targets, there are some disadvantages, including the non-facile preparation, difficulties in uniform dispersion, and toxicity of some of these nanomaterials. Therefore, it can be anticipated that the search for new nanomaterials is aimed towards replacements.

Metal-organic frameworks $(\mathrm{MOFs})^{3}$ are a novel class of crystalline material. They can show remarkable properties, such as large surface areas, structure tunability, and good aqueous dispersibility. Nevertheless, it cannot be ignored that MOFs still have some defects, restricting their application to a great extent. For instance, MOFs exhibit inferior performance in terms of electrical conduction and have poor stability. Encouragingly, there has been the idea of assembling MOFs with polymer materials to generate highly stable MOF-based bio-polymerized materials, called metal-phenolic nanomaterials (MPNs), which can combine the advantages of both parent materials. Since MPNs are superior to MOFs synthesized via traditional methods, they can be excellent alternatives to single MOFs to overcome the above-mentioned difficulties. MPNs have unique surface properties, including the ability to tune the particle size, surface groups, charges, structure, and central metal species. Moreover, MPNs exhibit strong $\pi-\pi$ stacking interactions (between exposed nitrogenous nucleobases and the phenolic rings of MPNs) ${ }^{22}$ and coordination interactions (between the DNA phosphate backbone and unsaturated metal sites of MPNs), ${ }^{23,24}$ highly enhancing the sensing performance.

Thus, we focused on the synthesis of a novel monoclinic-like metal-phenolic nanomaterial (MPN), which is a polymeric material involving tannic acid (TA). It has received increasing attention due to its high fluorescence quenching abilities, high surface area, tailorable composition, and diverse advantages, which include biological compatibility, sensing abilities, and environmental applications. Polyphenol is naturally present in vegetables, fruits, etc. TA is composed of a central glucose core surrounded by covalently linked digalloyl ester groups with a huge amount of phenolic hydroxyl groups. ${ }^{25,26}$ The preparation of NMc-CuTA involves the use of a plant-based polyphenol (TA) as organic ligands and metal species $\left(\mathrm{Cu}^{2+}\right)$. The polydentate ligand TA could be easily coordinated with metal ions $\left(\mathrm{Cu}^{2+}\right)$ to form a metal-TA $\left(\mathrm{Cu}^{2+}-\mathrm{TA}\right)$ complex. The surface of NMc-CuTA is rich in $\mathrm{sp}^{2}$-hybridized carbon atoms and $\mathrm{Cu}^{2+}$ ions with a paramagnetic quenching nature. This aspect is beneficial for increasing the $\pi-\pi$ and metal-ligand interactions between LFGr-ssDNA, AO, and NMc-CuTA. Therefore, LFGr-ssDNA and AO can be adsorbed on the NMc-CuTA surface via $\pi-\pi$ stacking and electrostatic interactions but, fortunately, double-stranded DNA (ds-DNA) and G-quadruplexes (G4 complexes) cannot be adsorbed onto NMc-CuTA due to the high shielding of nucleobases within the negatively charged phosphate backbone of dsDNA and G4 complexes. Based on these properties, NMcCuTA has been utilized to develop a new sensing system for the detection of heavy metal ions and biomolecules.

In this work, we report a novel NMc-CuTA- and LFGr-ssDNAbased fluorescent biosensor with superior sensitivity using the formation of NMc-CuTA/LFGr-ssDNA/AO for sensing $\mathrm{Pb}^{2+}$ and the drug Epn. AO is chosen as an excellent fluorescent indicator because this dye emits strong fluorescence in solution. LFGrSSDNA and AO bind to the surface of NMc-CuTA via stacking interactions. At the same time, when $\mathrm{Pb}^{2+}$ ions are introduced to the NMc-CuTA/LFGr-ssDNA/AO complex, then LFGr-ssDNA is involved in forming G4 complexes and evacuates from the surface of NMc-CuTA, leading to enhanced AO fluorescence intensity. However, the further addition of Cys, which is a highly effective reactant towards $\mathrm{Pb}^{2+}$, results in the formation of a $\mathrm{Pb}^{2+}$-Cys complex, which leads to free LFGr-ssDNA and AO. Now, this free LFGr-ssDNA and AO can again be adsorbed onto the NMc-CuTA surface, causing the fluorescence signal to decrease. When Epn is introduced into the quenched solution, it strongly intercalates with LFGr-ssDNA to form a G4 complex once again, which leads to the enhancement of the fluorescence intensity of AO. Thus, a novel Turn-ON fluorescence-signalbased label-free biosensor for the sequential detection of $\mathrm{Pb}^{2+}$ and Epn was developed, and this has not been reported by any other researchers to date.

\section{Experimental section}

\subsection{Materials and methods}

Tannic acid (TA) and copper nitrate trihydrate were purchased from Marcklin Biochemical Co., Ltd. Pluronic F127 was purchased from Sigma-Aldrich. The $\mathrm{Pb}^{2+}$ and Epn molecule binding DNA probe was synthesized using integrated DNA technology. The following DNA sequence was used: $5^{\prime}$ CCTGGGCGGGTAGGGCGGGATCGGGTCCAGGT. A stock solution of DNA was prepared via dissolving $50 \mathrm{mM}$ DNA in Tris hydrochloride (Tris- $\mathrm{HCl}$ ) buffer solution $(\mathrm{pH}=7.5)$ and it was stored at $4{ }^{\circ} \mathrm{C}$. Epirubicin, lead nitrate, mercury nitrate, calcium chloride, nickel chloride, cobalt chloride, copper chloride, barium chloride, iron chloride, magnesium chloride, manganese chloride, imatinib (IMT), ampicillin (AMP), streptomycin (STR), tamoxifen (TMF), and other standard chemicals were purchased from Sigma-Aldrich (India) and SRL Pvt Ltd (India). The stock buffer and metal salt solutions used in the experiments were prepared using double-distilled water.

\subsection{Structural characterization}

The fluorescence emission spectra were measured using a HORIBA JOBIN YVON Fluoromax-4 spectrofluorometer with a xenon lamp excitation source at excitation and emission wavelengths of 490 and $530 \mathrm{~nm}$, respectively, and a slit width of $5 \mathrm{~nm}$. The morphological appearance of NMc-CuTA was studied based on surface images obtained using a high-resolution scanning electron microscope (HRSEM, Nanosem 430) and 
high-resolution transmission electron microscope (HRTEM) at a voltage of $200 \mathrm{kV}$ with JEOL/JEM-2100 apparatus. Element mapping was also conducted with a Nanosem 430 HRSEM equipped with an energy dispersive X-ray spectrometer (EDX) (ISIS300; Oxford) to identify the distributions of $\mathrm{Cu}^{2+}$ ions, carbon, and oxygen on the surface of NMc-CuTA. An Agilent Technologies FT-IR spectrometer (USA) was used to record the Fourier-transform infrared (FT-IR) spectra via the $\mathrm{KBr}$ pellet technique. FT-IR spectroscopy was used to investigate the functional groups on the surface of NMc-CuTA in the range of 400-1400 $\mathrm{cm}^{-1}$. X-ray diffraction (XRD) spectra were obtained using a PAN analytical X'pert pro X-ray diffractometer with $\mathrm{Cu}$ $\mathrm{K} \alpha$ radiation. $\mathrm{XRD}$ patterns were used to find out the crystalline nature of the synthesised NMc-CuTA, and they were recorded in the $2 \theta$ range of $10-80^{\circ}$. X-ray photoelectron spectroscopy (XPS, PerkinElmer Phi 1600 ESCA system), using Mg (1486.6 eV) as the radiation source, was used to estimate the surface element composition, element binding configuration, and charge compensation of NMc-CuTA.

\subsection{Analysis of real clinical samples (RCS)}

For the quantitative analysis of $\mathrm{Pb}^{2+}$ and Epn in RCS, urine samples were filtered through a membrane to remove all insoluble impurities. Aliquots of the urine samples were spiked with a stock solution of $\mathrm{Pb}^{2+}$ and Epn diluted with Tris-HCl buffer. Then, fluorescence measurements were performed.

\subsection{Procedure for $\mathrm{Pb}^{2+}$ and Epn detection}

The detailed procedure for the detection of $\mathrm{Pb}^{2+}$ and Epn is as follows. Initially, the incorporated reaction was performed by mixing $500 \mu \mathrm{L}$ of $300 \mathrm{nM}$ LFGr-ssDNA and AO, each made with Tris buffer ( $\mathrm{pH} 7.5$ ), followed by the addition of $30 \mu \mathrm{g} \mathrm{mL} \mathrm{m}^{-1}$ NMc-CuTA into a micro-centrifuge tube. After $20 \mathrm{~min}$ of reaction, the solution was transferred into a cuvette and fluorescence spectra were measured at excitation and emission wavelengths of 490 and $530 \mathrm{~nm}$, respectively, showing a fall in AO fluorescence due to the quenching behavior of NMc-CuTA. Later, freshly prepared $\mathrm{Pb}^{2+}$ solution at the desired concentration was added and incubated for $30 \mathrm{~min}$ at $35^{\circ} \mathrm{C}$, leading to the formation of a $\mathrm{G} 4$ complex, which results in the enhancement of the fluorescence intensity of AO. Furthermore, this sensing strategy was prolonged for the subsequent detection of Epn via the elimination of $\mathrm{Pb}^{2+}$ using Cys, leading to the turn-off of the fluorescence signal. Finally, the desired concentration of Epn was added into the above solution, which was incubated for $30 \mathrm{~min}$ at $35{ }^{\circ} \mathrm{C}$, again leading to the formation of the $\mathrm{G} 4$ complex and causing an increase in AO fluorescence intensity.

\subsection{Synthesis of NMc-CuTA}

The novel monoclinic nanomaterial was synthesized according to the literature with some modifications. NMc-CuTA was prepared via a metal-ligand-coordination-driven self-assembly process. $^{27}$

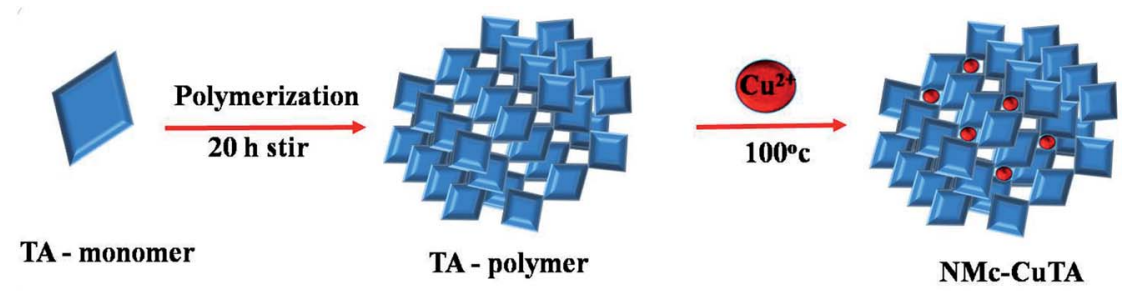

Scheme 1 A schematic representation of the synthesis of NMc-CuTA.
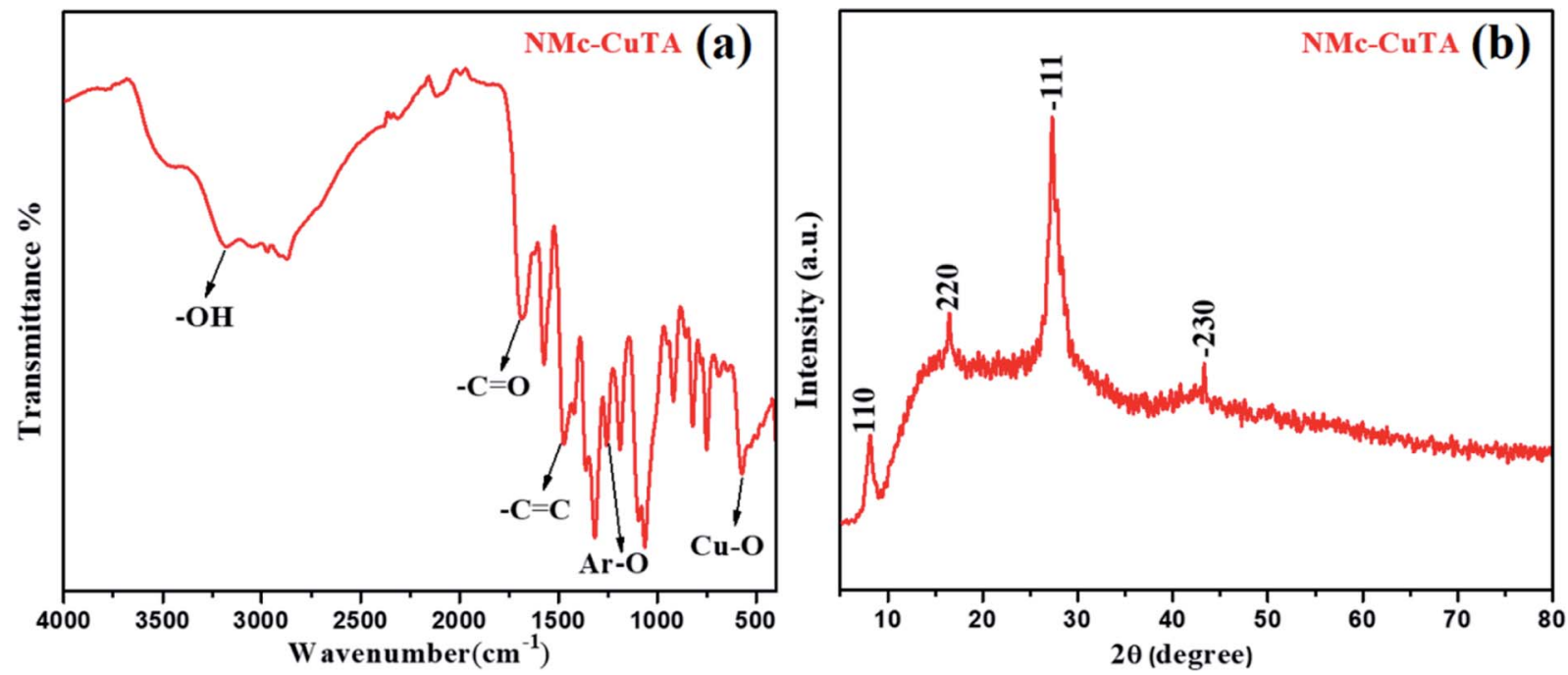

Fig. 1 (a) FT-IR and (b) XRD spectra of NMc-CuTA. 
Briefly, $500 \mathrm{mg}$ of $\mathrm{F} 127$ was dissolved in a mixture of water $(5 \mathrm{~mL})$ and ethanol $(10 \mathrm{~mL})$. Then, $900 \mu \mathrm{L}$ of ammonia was slowly added followed by the addition of tannic acid (500 $\mathrm{mg}$ ), and the above solution was magnetically stirred for $20 \mathrm{~h}$. To this, $250 \mathrm{mg}$ of $\mathrm{Cu}\left(\mathrm{NO}_{3}\right)_{2} \cdot 3 \mathrm{H}_{2} \mathrm{O}$ dissolved in a minimum volume of water was added, followed by stirring for $24 \mathrm{~h}$. The formed product was transferred into a pressure tube and placed in an oil bath for $15 \mathrm{~h}$ at $100{ }^{\circ} \mathrm{C}$. Further, the solution was centrifuged and washed several times with water and ethanol to remove unreacted materials. Finally, the obtained NMc-CuTA material was dried at $70{ }^{\circ} \mathrm{C}$ for $7 \mathrm{~h}$, with the synthesis process shown in Scheme 1 .

\section{Results and discussion}

\subsection{Characterization}

In order to confirm the structural transformation of the prepared NMc-CuTA, a few studies were carried out. Initially,
FT-IR spectral studies were done, and the results are shown in Fig. 1a. It is shown that the high intensity peak at $3172 \mathrm{~cm}^{-1}$ corresponds to the typical -OH stretching frequency, the peak at $3080 \mathrm{~cm}^{-1}$ can be assigned to $-\mathrm{C}-\mathrm{H}$ aromatic ring stretching, and the peak at about $1693 \mathrm{~cm}^{-1}$ is due to the $\mathrm{C}=\mathrm{O}$ stretching of the carbonyl group. Further, the specific absorption peak located at $1470 \mathrm{~cm}^{-1}$ was attributed to $\mathrm{C}=\mathrm{C}$ aromatic ring stretching, and the peak at $1270 \mathrm{~cm}^{-1}$ corresponds to $\mathrm{Ar}-\mathrm{O}$ (phenolic) groups. Furthermore, the peak appeared below $600 \mathrm{~cm}^{-1}$ is attributed to $\mathrm{Cu}-\mathrm{O}$ vibrations; this clearly indicates successful bonding with copper atoms on the surface of tannic acid. ${ }^{28,29}$ The $-\mathrm{OH}$ frequency reduction from $3500 \mathrm{~cm}^{-1}$ to $3172 \mathrm{~cm}^{-1}$ gives clear evidence that tannic acid will form a complex with copper metal ions. The above results indicate the presence of numerous $-\mathrm{C}=\mathrm{C}$ and phenolic groups on the surface of NMc-CuTA along with covalently bonded copper metal ions. The above fact suggests that the prepared NMc-
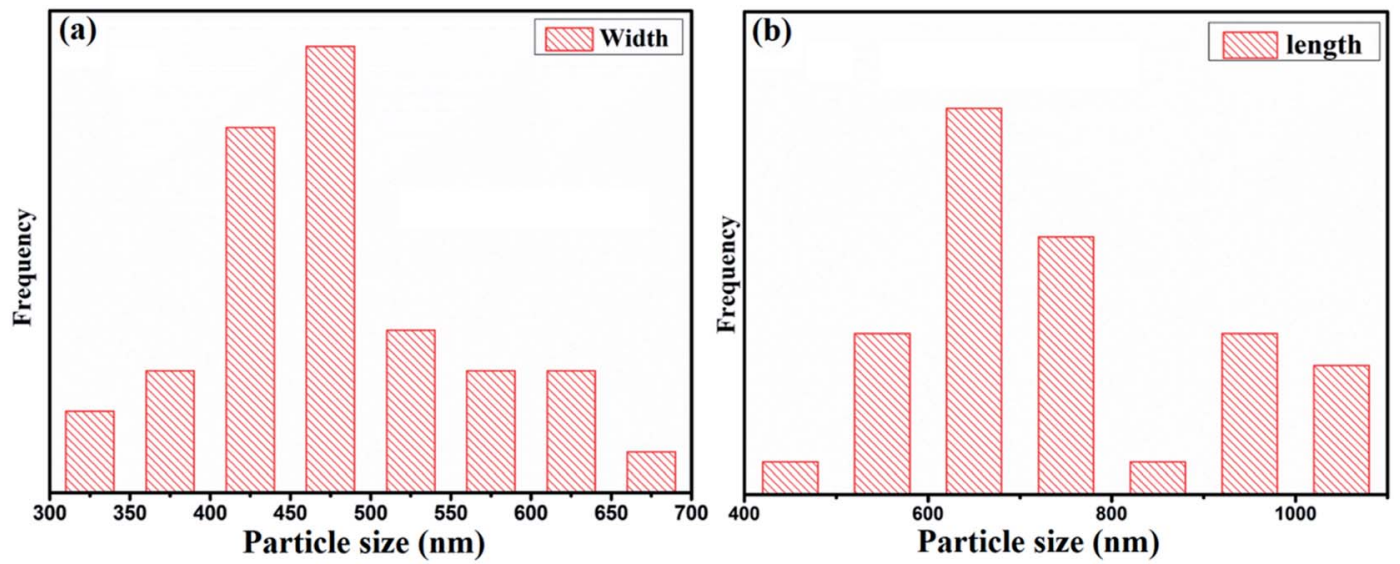

Fig. 2 Particle sizes of NMc-CuTA: (a) width and (b) length.

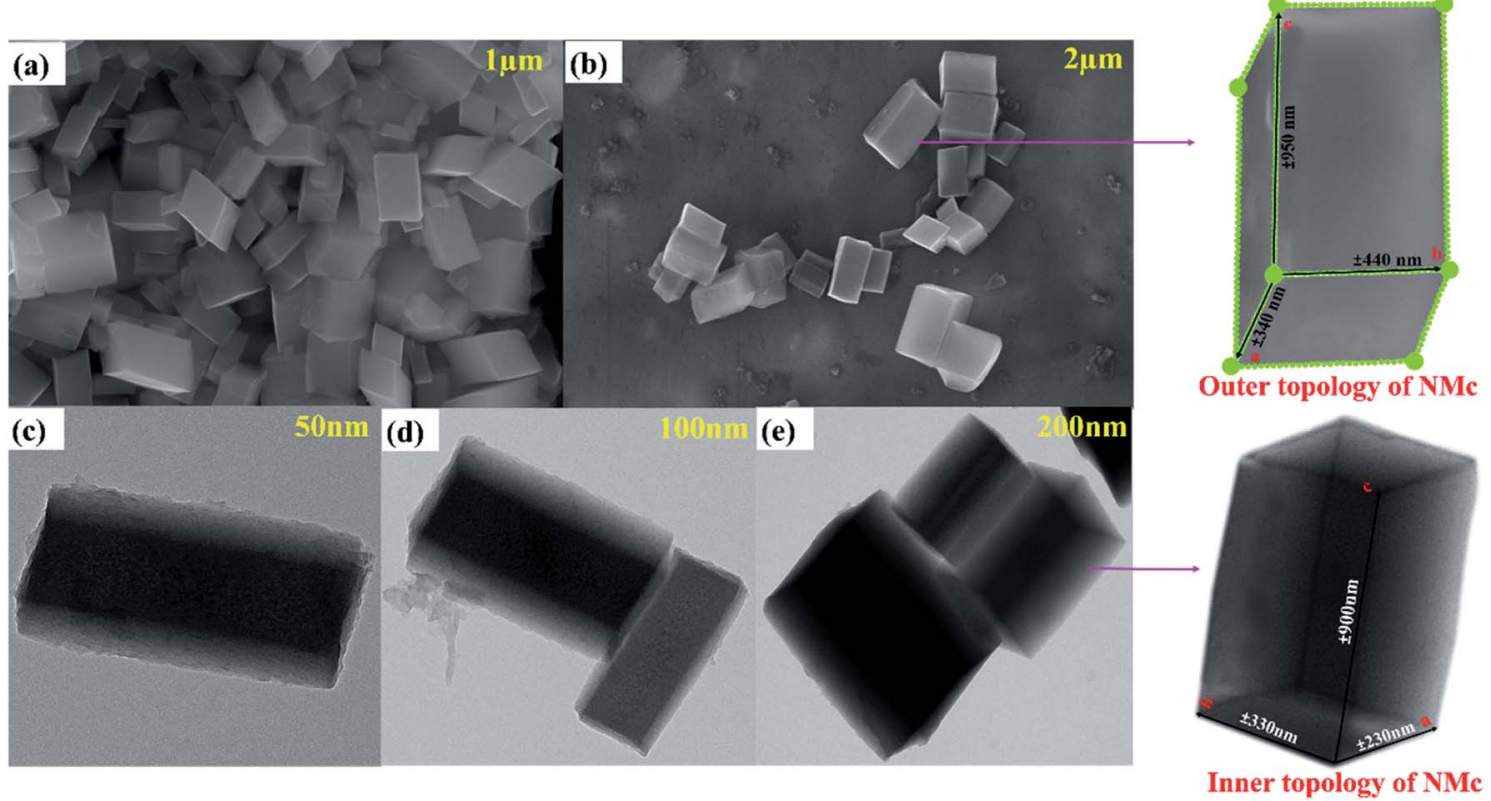

Fig. 3 ( $a$ and b) HRSEM and (c-e) HRTEM images of NMc-CuTA. 


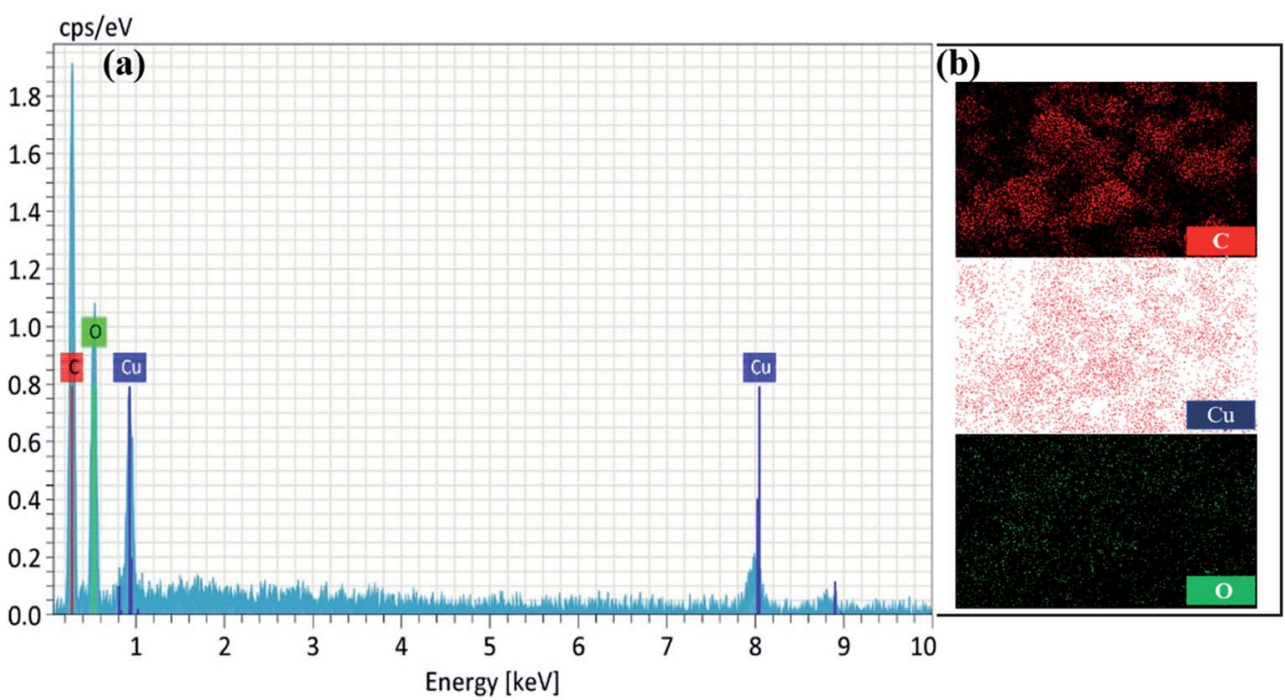

Fig. 4 EDX spectrum (a) and elemental mapping (b) of NMc-CuTA.

CuTA complex can show greatly enhanced hydrophilicity and stability in aqueous solution. As shown in Fig. 1b, the XRD diffraction peaks located at $2 \theta$ values of about 8.2, 16.4, 27.1, and $43.0^{\circ}$ could be ascribed to the (110), (220), $(-111)$, and $(-230)$ crystal planes, respectively, which might suggest a monoclinic copper-tannic acid lattice. In particular, the peak at a $2 \theta$ value of $43.0^{\circ}$ was assigned to the copper $(-230)$ plane (PDF no. 00-032-0331). ${ }^{30}$

The morphology and elemental analysis of the synthesized metal-phenolic (copper-tannic acid) nanomaterial were studied via SU-70 high-resolution scanning electron microscopy along with X-ray energy dispersive spectrometry (EDS). The HRSEM image confirms that the synthesized copper-tannic acid particles possess a monoclinic shape (Fig. 3a and b). Thus, the material was named "nano-monoclinic copper tannic acid" (NMc-CuTA). As NMc-CuTA has a large surface area, it can adsorb LFGr-ssDNA and AO efficiently due to $\pi-\pi$ stacking, which in turn quenches the fluorescence of $\mathrm{AO}$ due to the paramagnetic quenching abilities of the $\mathrm{Cu}^{2+}$ ions. HRSEM
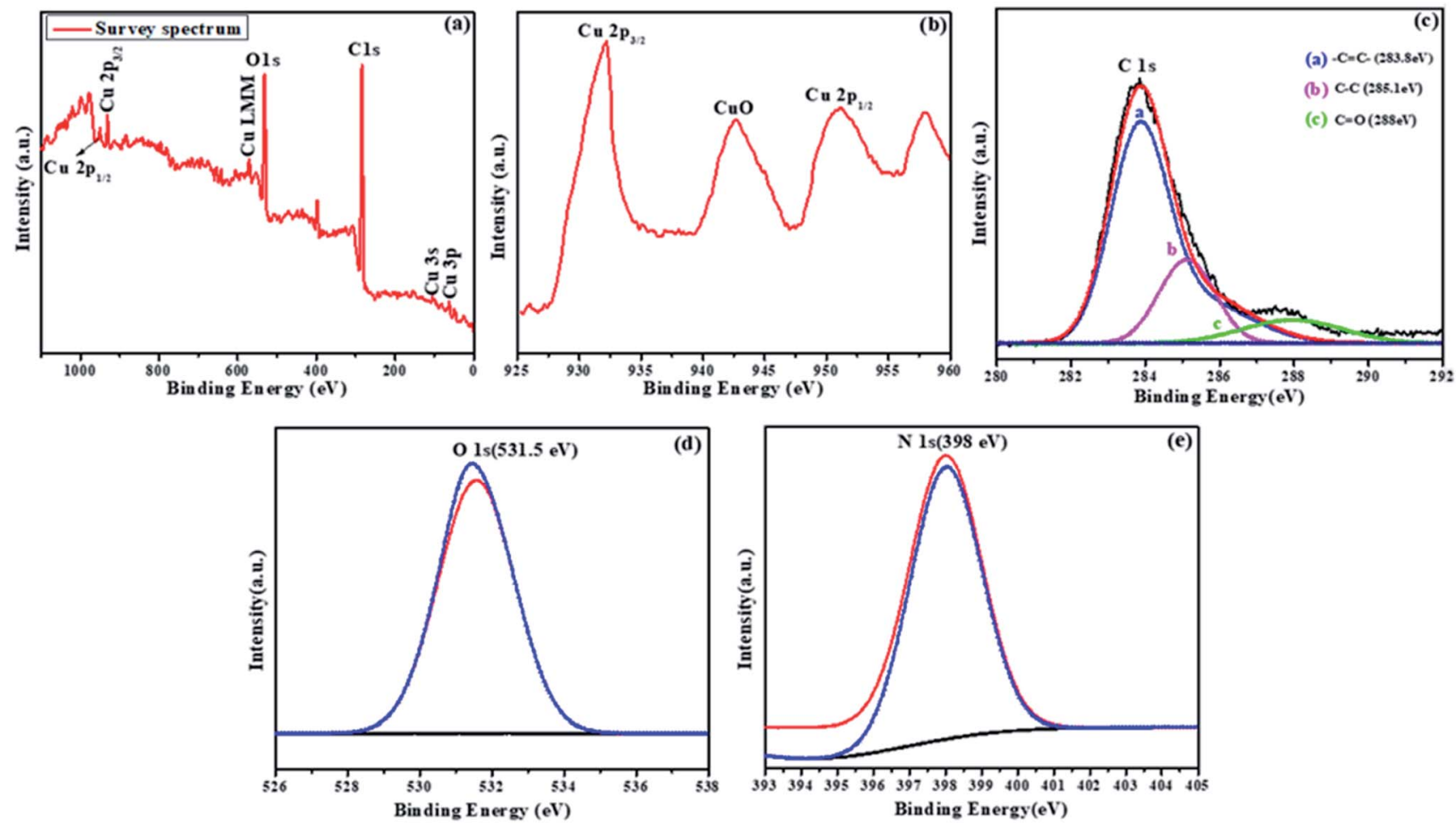

Fig. 5 XPS spectra of NMc-CuTA: (a) survey, (b) Cu 2p, (c) C 1s, (d) O 1s, and (e) N 1s spectra. 


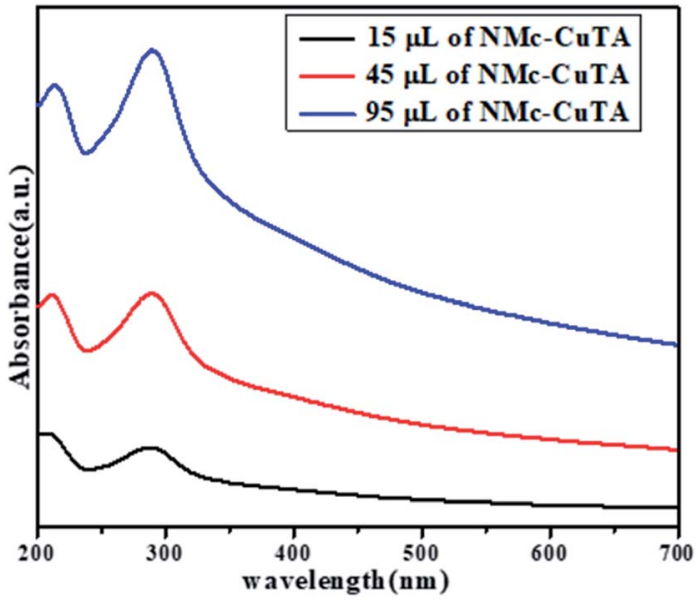

Fig. 6 UV-spectra of different amounts of NMc-CuTA.

images show that typical NMc-CuTA particles have a width of $\sim 450 \mathrm{~nm}$ and a length of $\sim 650 \mathrm{~nm}$, as shown in Fig. $2 \mathrm{a}$ and $\mathrm{b}$.

Furthermore, a clear inner surface investigation of the asprepared nanomaterial was carried out via HRTEM, as shown in Fig. 3c-e. HRTEM images show that the lengths of the unit cell parameters obtained were in the order of $a \neq b \neq c(a \pm$ $230 \mathrm{~nm} ; b \pm 330 \mathrm{~nm}$ and $c \pm 900 \mathrm{~nm}$ ), which clearly expresses that the $\mathrm{Cu}^{2+}$ ions are coordinated to the surface of tannic acid with monoclinic geometry. The EDX spectrum and elemental mapping of NMc-CuTA are shown in Fig. 4a and b. The EDX spectrum confirms the presence of the elements carbon (C), oxygen $(\mathrm{O})$, and copper $(\mathrm{Cu})$ with atomic percentages of 45,50 , and 5, respectively. This result is consistent with the XPS data. The elemental mapping of this nanomaterial also reveals the presence of the elements $\mathrm{C}, \mathrm{O}$, and $\mathrm{Cu}$, marked in different colours. The elemental mapping and EDX results conclude that the synthesized nanomaterial was found to be free from any other elements or impurities.
To confirm the purity and elemental composition of the prepared NMc-CuTA material, X-ray photoelectron spectroscopy (XPS) measurements were performed, as seen in Fig. 5a-e. As shown in Fig. 5a, the spectrum of NMc-CuTA confirmed the presence of $\mathrm{C} 1 \mathrm{~s}, \mathrm{O} 1 \mathrm{~s}, \mathrm{~N} 1 \mathrm{~s}$, and $\mathrm{Cu} 2 \mathrm{p}$ peaks, without any impurities. The peaks found at 950.1 and $932.1 \mathrm{eV}$ in the $\mathrm{Cu} 2 \mathrm{p}$ XPS spectrum are attributed to $\mathrm{Cu} 2 \mathrm{p}_{1 / 2}$ and $\mathrm{Cu} 2 \mathrm{p}_{3 / 2}$, respectively (Fig. 5b). The high-resolution $\mathrm{C}$ 1s spectrum was fitted with three peaks at about 283.8, 285.1, and $288 \mathrm{eV}$, which could be assigned to $\mathrm{C}=\mathrm{C}, \mathrm{C}-\mathrm{C}$, and $\mathrm{C}=\mathrm{O}$ or $\mathrm{C}-\mathrm{O}$, respectively, with relative amounts of $48 \%, 30 \%$, and $12 \%$, respectively ${ }^{31}$ (Fig. $5 \mathrm{c}$ ). The O 1s XPS spectrum (Fig. 5d) exhibits a peak at $531.5 \mathrm{eV}$, which was assigned to the lattice oxide oxygen of the metal oxide, indicating the formation of metal-oxygen $(\mathrm{Cu}-\mathrm{O})$ bonds between copper and tannic acid. The N 1s XPS spectrum has a single intense peak at $398 \mathrm{eV}$, which corresponds to the $\mathrm{N}$ atoms of ammonia (Fig. 5e). Therefore, with the help of XPS data, we conclude that NMc-CuTA has ionic copper and a large amount of $\mathrm{sp}^{2}$-hybridized carbon; it can effectively diminish the fluorescence properties of AO and facilitate the adsorption of LFGr-ssDNA on its surface via $\pi-\pi$ stacking interactions.

$\mathrm{N}_{2}$ adsorption-desorption studies are used to find the surface area of NMc-CuTA, and results are shown in Fig. S1. $\dagger$ The BET surface area and pore volume of NMc-CuTA are found to be $178.692 \mathrm{~m}^{2} \mathrm{~g}^{-1}$ and $0.299 \mathrm{~cm}^{3} \mathrm{~g}^{-1}$, respectively. From these observations, polyphenol-derived NMC-CuTA is found to have a high surface area and pore volume. Therefore, NMCCuTA had high adsorption abilities toward ssDNA and AO due to its high surface area.

\subsection{The quenching behavior of NMc-CuTA and confirmation studies}

The optical properties of NMc-CuTA were investigated via UV-vis spectroscopy in the range of $200-800 \mathrm{~nm}$. In general, the UV spectrum of tannic acid shows two absorption peaks at 210 and $280 \mathrm{~nm}$, which are attributed to $\pi-\pi^{*}$ and $\mathrm{n}-\pi^{*}$ transitions,

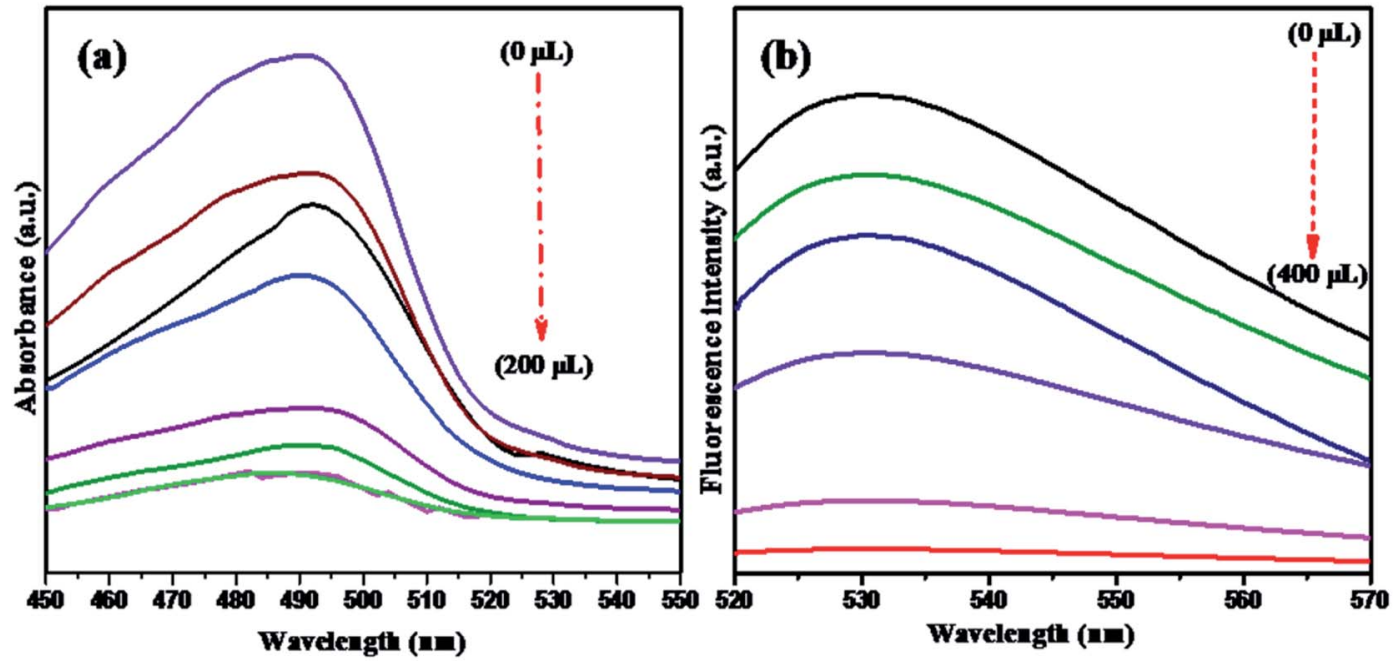

Fig. 7 (a) UV-visible spectra and (b) fluorescence spectra showing the quenching of AO in the presence of various amounts of NMc-CuTA. (a) AO treated with $0,50,75,100,150,175,200$, and $225 \mu \mathrm{L}$ of NMc-CuTA; and (b) AO treated with 0, 50, 100, 200, 300, and 400 $\mu \mathrm{L}$ of NMc-CuTA. 
respectively, ${ }^{32}$ and the $\mathrm{Cu}^{2+}$ ion absorption peak is found around $275 \mathrm{~nm}$. Due to bond formation between copper and tannic acid, the absorption peaks at 280 and $275 \mathrm{~nm}$ merge to give a new absorption peak at $290 \mathrm{~nm}$, as shown in Fig. 6 .

NMc-CuTA has good fluorescence quenching abilities, as shown in Fig. 7. The UV absorption spectrum of AO shows a strong absorption band at $490 \mathrm{~nm}$. The absorption band intensity decreases with respect to the amount of NMc-CuTA (Fig. 7a). As the absorption band has high hypochromicity, we conclude that AO and NMc-CuTA undergo strong static interactions due to the large amount of $\mathrm{sp}^{2}$-hybridized carbon atoms. This may also be due to the paramagnetic quenching characteristics of copper(II) ions present on the surface of NMcCuTA.

The fluorescence emission spectra of $\mathrm{AO}$ in the presence and absence of NMc-CuTA were studied. As the amount of NMc-
CuTA is increased, the fluorescence emission intensity of AO gradually decreases due to intermolecular energy transfer between AO and NMc-CuTA. This action requires large overlap between the emission spectrum of the energy donor $\mathrm{AO}$ and the absorption spectrum of the energy acceptor NMc-CuTA, as this is a basic necessity for the FRET process. Thus, the mechanism behind the strong fluorescence quenching effect of NMc-CuTA toward AO could be concluded, as shown in Fig. 7b.

\subsection{The sensing mechanism toward $\mathrm{Pb}^{2+}$ ions and Epn}

In the designed strategy, the mechanism proposed allowed a Turn-ON fluorometric biosensor for $\mathrm{Pb}^{2+}$ and $\mathrm{Epn}$ with superior sensitivity via the quenching of the AO fluorophore by the NMc-CuTA/LFGr-ssDNA complex, as shown in Scheme 2. The single-stranded DNA was designed with a large extended Grich sequence, which is utilized as the specific target analyte.
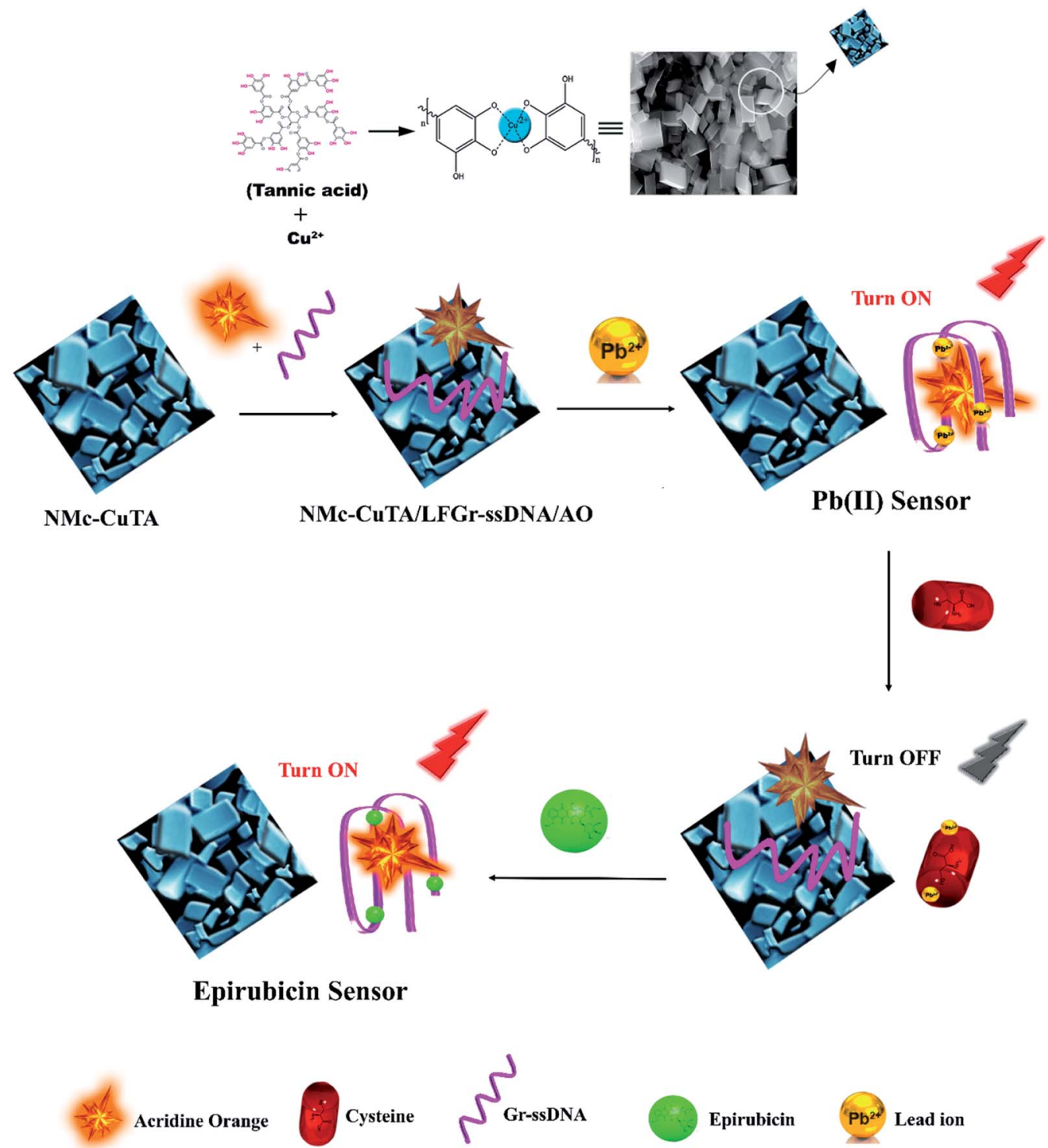

Scheme 2 A schematic illustration of the fluorescence detection of $\mathrm{Pb}^{2+}$ ions and the anti-cancer drug Epn using the NMc-CuTA/LFGr-ssDNA/ AO sensing platform. 


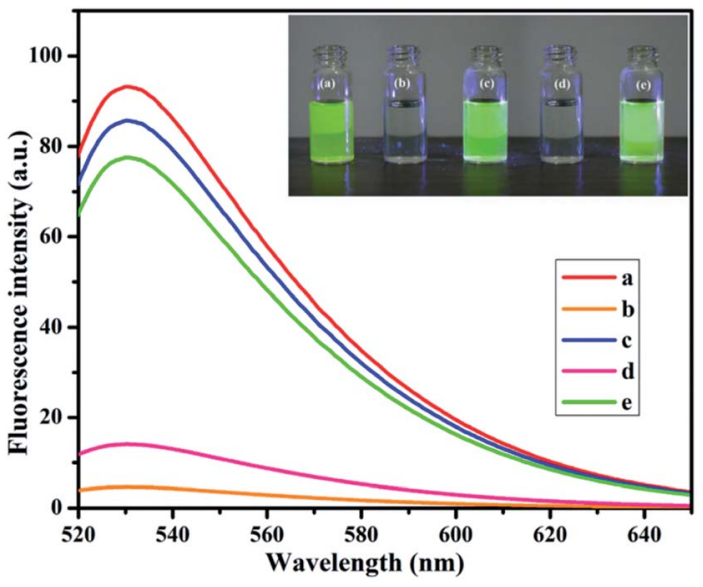

Fig. 8 The fluorescence emission spectra and UV properties (inset) of AO alone (a), the NMc-CuTA/LFGr-ssDNA/AO complex (b), the NMcCuTA/Pb ${ }^{2+}-\mathrm{AO}-\mathrm{G} 4$ complex (c), the NMc-CuTA/AO/LFGr-ssDNA/ $\mathrm{Pb}^{2+}$-Cys complex (d), and the NMc-CuTA/Epn-AO-G4 complex/ $\mathrm{Pb}^{2+}$-Cys complex (e).

LFGr-ssDNA binds to the surface of NMc-CuTA through $\pi-\pi$ stacking and electrostatic interactions. $\mathrm{AO}$ is an excellent fluorescent indicator because this dye emits strong fluorescence in solution (Fig. 8a). The fluorescence of AO could be quenched with NMc-CuTA in end-stacking mode to form the NMc-CuTA/ LFGr-ssDNA/AO complex, as shown in Fig. 8b, resulting in a significant decrease in the $\mathrm{AO}$ fluorescence signal due to the paramagnetic quenching nature of $\mathrm{Cu}^{2+}$ via fluorescence resonance energy transfer (FRET) from the fluorophore singlet excited state $\left(\mathrm{S}_{\mathrm{n}}\right)$ to the paramagnetic $\mathrm{Cu}^{2+}$ center. At the same time, $\mathrm{Pb}^{2+}$ ions introduced into the NMc-CuTA/LFGr-ssDNA/AO complex form a $\mathrm{Pb}^{2+}-\mathrm{G} 4$ complex, leading to enhanced fluorescence intensity (Fig. 8c) because the G4 complex strongly captures AO from the surface of NMc-CuTA. The further addition of cysteine, as a highly effective reactant towards $\mathrm{Pb}^{2+}$, results in the formation of $\mathrm{Pb}^{2+}$-Cys complexes, leading to the generation of free LFGr-ssDNA and AO, which again are adsorbed onto the NMc-CuTA surface with a reduced fluorescence signal in turn. When Cys is added to the $\mathrm{AO} / \mathrm{Pb}^{2+}-\mathrm{G} 4$ complex, it efficiently attracts $\mathrm{Pb}^{2+}$ ions from the $\mathrm{G} 4$ complex to form more stable $\mathrm{Pb}^{2+}$-Cys complexes, as biothiol-like Cys contains active functional groups such as $-\mathrm{SH},-\mathrm{NH}_{2}$, and $-\mathrm{COOH}$. These groups strongly react with $\mathrm{Pb}^{2+}$ (based on HSAB theory), resulting in the precipitation of $\mathrm{PbS}$ or complexation via covalent forces, electron sharing, or exchange between the active functional group and $\mathrm{Pb}^{2+}$ ions. In the absence of $\mathrm{Pb}^{2+}$ ions, the G4 complex is unstable, so it aligns itself with unstructured DNA. Due to the absence of the G4 complex in solution, AO itself gets adsorbed on the NMc-CuTA surface, which significantly decreases the fluorescence intensity of $\mathrm{AO}$, as shown in Fig. 8d. When epirubicin (Epn), an anti-cancer drug, is added to the above solution, it strongly intercalates with the G-bases of unstructured DNA. This intercalation leads to the formation of hydrogen and covalent bonds between Epn and DNA guanine base pairs. These interactions can be stabilized by a covalent bond mediator, say cellular formaldehyde, resulting in the

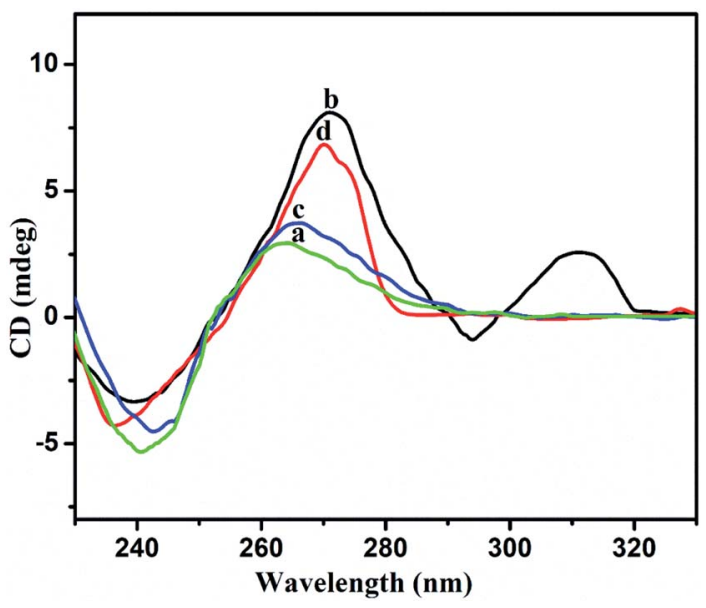

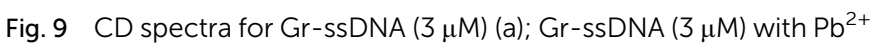
$(5 \mu \mathrm{M})(\mathrm{b})$; $\mathrm{Gr}$-ssDNA $(3 \mu \mathrm{M})$ with $\mathrm{Pb}^{2+}(5 \mu \mathrm{M})$ and Cys $(5 \mu \mathrm{M})$ (c); and $\mathrm{Gr}$ ssDNA $(3 \mu \mathrm{M})$ with $\mathrm{Pb}^{2+}(5 \mu \mathrm{M})$, Cys $(4 \mu \mathrm{M})$, and Epn $(5 \mu \mathrm{M})(\mathrm{d})$.

formation of Epn-G4 complexes, which can also capture AO from the surface of NMc-CuTA, leading to fluorescence signal recovery (Fig. 8e).

\subsection{Analysis of CD spectra}

The structural changes were further analyzed via circular dichroism (CD) spectroscopy. CD is a method that is quite sensitive to the confirmations of chiral structures, nucleic acids, and G-quadruplex structures. CD spectral analysis for $3 \mu \mathrm{M} \mathrm{Gr}-$ ssDNA in the absence and presence of $\mathrm{Pb}^{2+}$ and Epn is shown in Fig. 9. In the absence of $\mathrm{Pb}^{2+}$ and Epn, the $\mathrm{CD}$ spectrum has a low amplitude, as ssDNA has a barely chiral structure (Fig. 9, curves a and c). When incubated in $5 \mu \mathrm{M} \mathrm{Pb}^{2+}$, the spectrum possess a positive $\mathrm{CD}$ peak near $311 \mathrm{~nm}$ coupled with a negative peak at $294 \mathrm{~nm}$, which are characteristic of an antiparallel G4 structure orientation (Fig. 9, curve b) ${ }^{33}$ On the other hand, a confirmational change of ssDNA occurs upon the addition of 5 $\mu \mathrm{M}$ Epn, and the CD spectrum shows a positive peak at $270 \mathrm{~nm}$ and a negative peak at $236 \mathrm{~nm}$ (Fig. 9, curve d). These two peaks correspond to the parallel orientation of the G4 structure. Based on these results, we can conclude that Epn strongly intercalates with ssDNA, ${ }^{20}$ which leads to the formation of Epn-stabilized parallel G4 structures.

\subsection{Optimization of the detection conditions}

The optimization of the proposed sensor was evaluated based on various influencing factors, which include the concentrations of LFGr-ssDNA and AO, the amount of NMc-CuTA, and the effects of the reaction time, $\mathrm{pH}$, and temperature on the signalto-background ratio (S/B ratio), as shown in Fig. 10. Initially, the AO fluorescence intensity of the sensing system was studied via varying the concentration of LFGr-ssDNA, because the amount of G4 complex formation depends on the concentration of LFGrssDNA. The AO emission intensity was enhanced to a maximum level due to an increasing amount of G4 complex formation with respective analytes $\left(\mathrm{Pb}^{2+}\right.$ and $\left.\mathrm{Epn}\right)$; therefore, the $\mathrm{AO}$ 

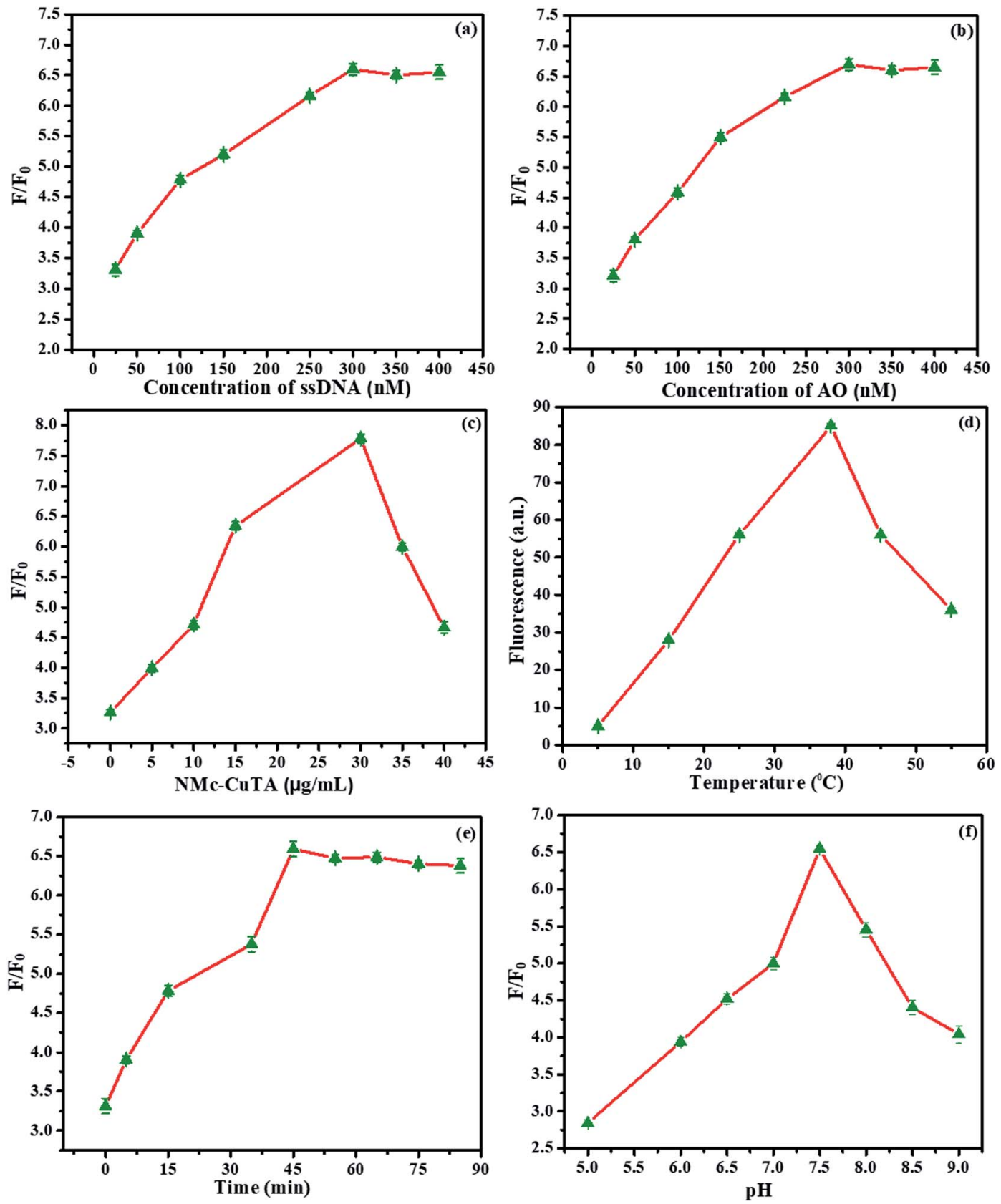

Fig. 10 Optimization of the detection conditions. (a) The effects of DNA concentration on fluorescence (different concentrations of LFGrssDNA were mixed with $30 \mu \mathrm{g} \mathrm{mL}^{-1} \mathrm{NMc}-\mathrm{CuTA}, 300 \mathrm{nM} \mathrm{AO}$, and $\mathrm{Pb}^{2+}(1 \mu \mathrm{M})$ or Epn $(1 \mu \mathrm{M})$ in $20 \mathrm{nM}$ Tris buffer). (b) The effects of AO on fluorescence (solutions of $20 \mathrm{mM}$ Tris buffer containing various concentrations of AO with LFGr-ssDNA (300 nM), $30 \mu \mathrm{g} \mathrm{mL}{ }^{-1} \mathrm{NMc}-\mathrm{CuTA}$, and $\mathrm{Pb}^{2+}(1 \mu \mathrm{M})$ or Epn $(1 \mu \mathrm{M})$ were used). (c) The fluorescence recovery abilities of NMc-CuTA (various amounts of NMc-CuTA, 300 nM AO, LFGrssDNA (300 nM), and $\mathrm{Pb}^{2+}(1 \mu \mathrm{M})$ or Epn $(1 \mu \mathrm{M})$ were mixed in $20 \mathrm{mM}$ Tris $\mathrm{HCl}$ buffer $(\mathrm{pH}=7.5)$, and then the AO fluorescence emission intensity was recorded). The effects of (d) temperature, (e) time, and (f) $\mathrm{pH}$ on the fluorescence recovery (20 mM Tris- $\mathrm{HCl}$ buffer solutions containing AO (300 nM), LFGr-ssDNA (300 nM), NMc-CuTA $\left(30 \mu \mathrm{g} \mathrm{mL}^{-1}\right)$, and $\mathrm{Pb}^{2+}(1 \mu \mathrm{M})$ or Epn $(1 \mu \mathrm{M})$ were used, with fluorescence spectra measured at different temperatures, times, and $\mathrm{pH}$ levels).

fluorescence emission indirectly depends on the concentration of LFGr-ssDNA. Accordingly, the concentration of LFGr-ssDNA was optimized between 25 and $400 \mathrm{nM}$, (Fig. 10a); the experimental results clearly show that fluorescence emission reached the maximum level when the concentration of LFGr-ssDNA was $300 \mathrm{nM}$. Therefore, the optimal concentration of LFGr-ssDNA (300 nM) was chosen as the fixed concentration for further studies. Similarly, the fluorophore concentration was optimised between 25 and $400 \mathrm{nM}$ (Fig. 10b). The experimental results show that the SBR of the AO signal reached a maximum with $300 \mathrm{nM}$ AO. Therefore, $300 \mathrm{nM}$ AO was chosen as the optimized concentration for this sensing system. Further, the amount of NMc-CuTA needs to be optimised, as the nanomaterial has the ability to quench the fluorescence intensity of AO. Similarly, the amount of NMc-CuTA was investigated in the presence of $300 \mathrm{nM}$ LFGr-ssDNA and $300 \mathrm{nM}$ AO. The experimental results 
show that the SBR of the AO signal decreases with an increasing amount of NMc-CuTA, and the AO signal intensity reached a minimum at an optimal concentration of $30 \mu \mathrm{g} \mathrm{mL}{ }^{-1}$; this also proves to be the optimal concentration for fluorescence recovery, as shown in Fig. 10c. Therefore, $30 \mu \mathrm{g} \mathrm{mL}{ }^{-1}$ was chosen as the optimised concentration for further studies.

The influence of the reaction temperature and incubation time on the performance of the sensing system was also optimized (Fig. 10d and e). The SBR of AO fluorescence increased to a maximum at $35{ }^{\circ} \mathrm{C}$. The incubation time was monitored from 0 to 90 minutes. At an incubation time of $45 \mathrm{~min}$, the fluorescence intensity reached a maximum value, so we chose $45 \mathrm{~min}$ as the optimal fluorescence recovery time. Therefore, further experiments were all conducted for $45 \mathrm{~min}$ at $35{ }^{\circ} \mathrm{C}$ for the detection of $\mathrm{Pb}^{2+}$ and Epn. Moreover, the influence of $\mathrm{pH}$ on the SBR of the AO signal was also optimized (Fig. 10f). The optimal $\mathrm{pH}$ was studied by using buffer solutions with different $\mathrm{pH}$ values; based on the results, a pH value of 7.5 was suitable for this sensing system.

\subsection{Sensitivity toward $\mathbf{P b}^{2+}$ and Epn}

To evaluate the presence of $\mathrm{Pb}^{2+}$ ions, a fluorescence spectra study was chosen. It was found that the fluorescence intensity was directly proportional to the concentration of detected $\mathrm{Pb}^{2+}$ ions using the above standard optimized conditions, as shown in Fig. 11. Herein, we explored the LFGr-ssDNA-based fluorescent $\mathrm{Pb}^{2+}$ sensor with improved sensitivity using AO dye adsorbed on the surface of NMc-CuTA with the LFGr-ssDNA complex. AO dye was chosen as the fluorescent indicator because this dye emits strong fluorescence in solution. In the absence of $\mathrm{Pb}^{2+}$ ions, there is no fluorescence signal because the fluorescence intensity was completely quenched by NMc-CuTA. In the presence of $\mathrm{Pb}^{2+}$, the fluorescence intensity of the NMcCuTA/LFGr-ssDNA/AO complex dramatically increased due to the formation of G4 complexes. This remarkable fluorescence intensity increased with an increase in the concentration of $\mathrm{Pb}^{2+}$ ions from 0 to $3000 \mathrm{nM}$, as seen in Fig. 11a and b. The relative fluorescence intensity was plotted against the concentration of $\mathrm{Pb}^{2+}$ ions in the range from 0 to $100 \mathrm{nM}$, and the linear correlation co-efficient $\left(R^{2}\right)$ was 0.9985 , as shown in Fig. 11c. The detection limit for $\mathrm{Pb}^{2+}$ ions was $1.5 \mathrm{nM}$, which was calculated based on the $3 \sigma /$ slope rule.

Further, the NMc-CuTA/ $\mathrm{Pb}^{2+}-\mathrm{G} 4-\mathrm{AO}$ system acts as another fluorescent assay for Epn detection. At first, $500 \mathrm{nM}$ Cys was added to the above system and it was incubated for $30 \mathrm{~min}$; the $\mathrm{Pb}^{2+}$ ions were strongly attracted by Cys to form a $\mathrm{Pb}^{2+}$-Cys complex, leading to a significant decrease in the fluorescence signal. Then, the introduced Epn intercalates with the G bases
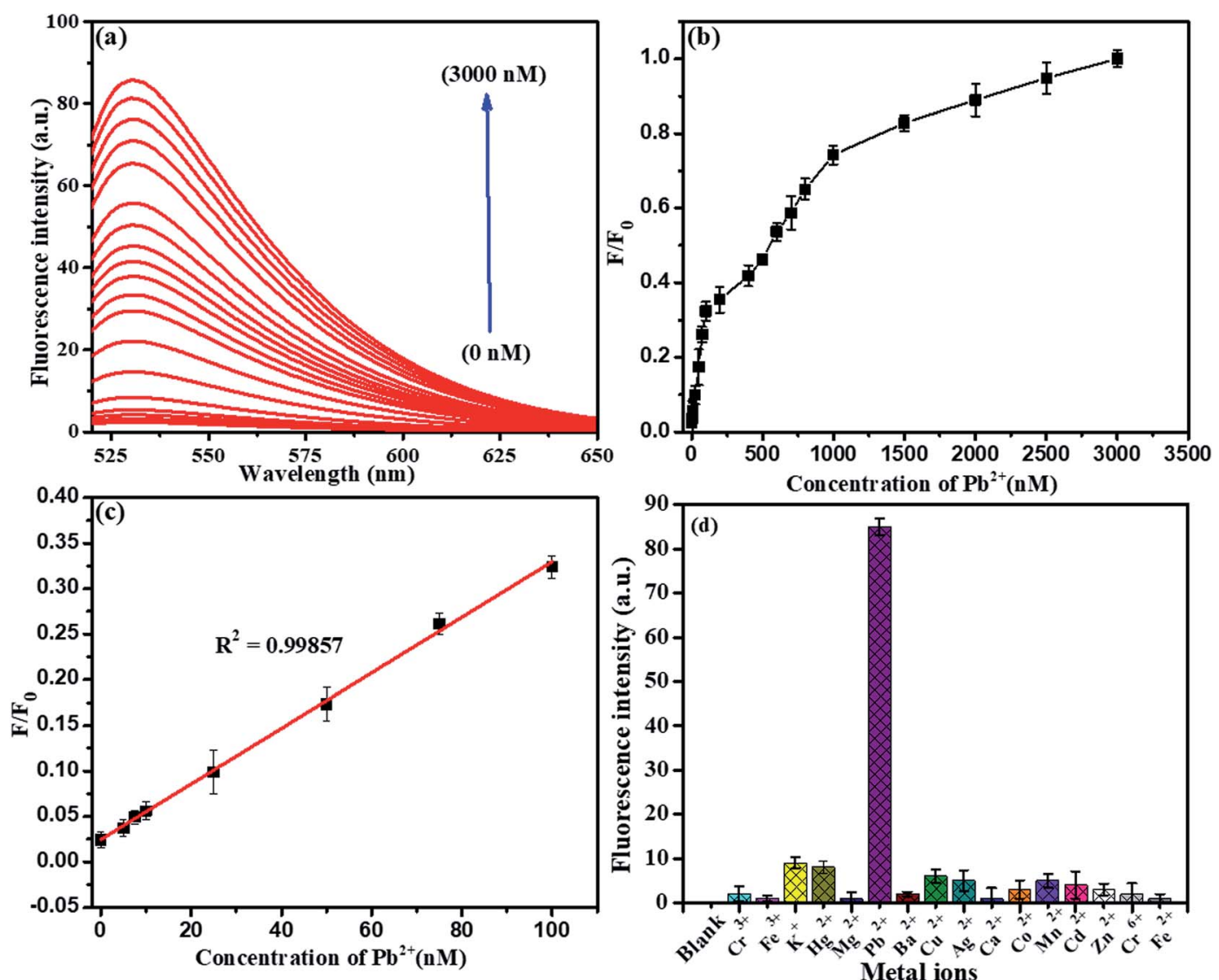

Fig. 11 (a) Fluorescence emission spectra of the optimized sensor in response to different concentrations of $\mathrm{Pb}^{2+}: 0,5,7.5,10,25,50,75,100$, $200,400,500,600,700,800,1000,1500,2000,2500$, and 3000 nM. (b) Integrated fluorescence response versus the Pb ${ }^{2+}$ concentration. (c) The linear relationship between the fluorescence emission intensity and $\mathrm{Pb}^{2+}$ concentration. (d) The selectivity of the optimized fluorescent sensor for $\mathrm{Pb}^{2+}(500 \mathrm{nM})$ in comparison to other metal ions $(1000 \mathrm{nM})$. The error bars display the standard deviations from three individual tests. 

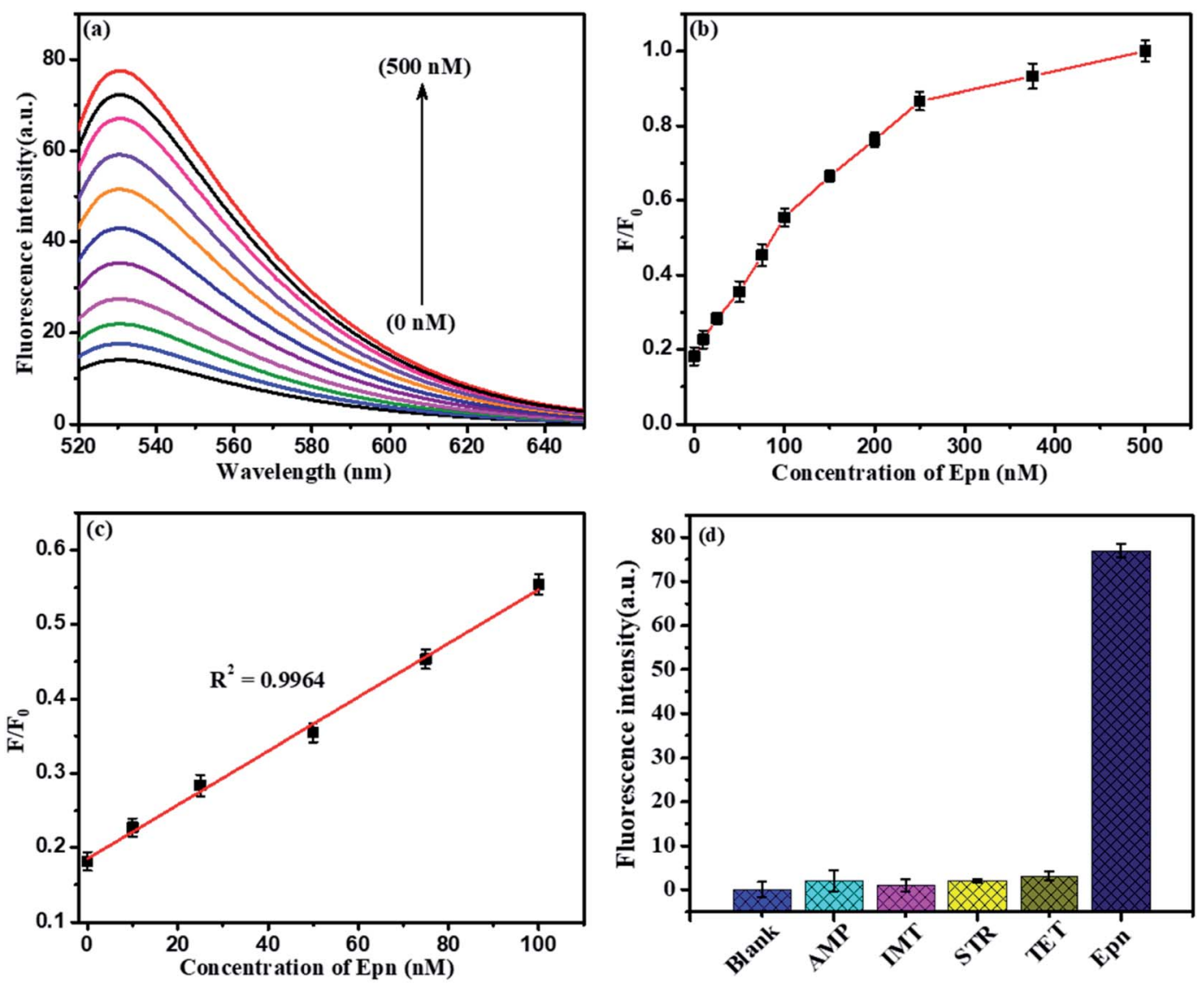

Fig. 12 (a) Fluorescence spectra of the optimized sensor in response to different concentrations of Epn: 0, 10, 25, 50, 75, 100, 150, 200, 250, 375, and $500 \mathrm{nM}$. (b) Integrated fluorescence response versus the Epn concentration. (c) The linear relationship between the fluorescence emission intensity and the Epn concentration. (d) The selectivity of the optimized fluorescence sensor for Epn (500 nM) in comparison to other drugs (1000 nM). The error bars display the standard deviations from three individual tests.

in ssDNA to form G4 complexes, which capture AO; the fluorescence enhancement was monitored in the range of 0 to $500 \mathrm{nM}$, as shown in Fig. 12a and b. A good linear relationship $\left(R^{2}=0.9964\right)$ was observed in the range from 0 to $100 \mathrm{nM}$ and the limit of detection (LOD) was $5.6 \mathrm{nM}$ (Fig. 12c). Furthermore, the proposed $\mathrm{Pb}^{2+}$ and Epn sensor showed superior performance compared to other LFGr-ssDNA-based methods. The high sensitivity of the proposed sensor can be attributed to the quenching ability of NMc-CuTA, indicating the formation of NMc-CuTA/LFGr-ssDNA/AO complexes, which efficiently act as sensing probes. The advantages of our proposed sensing probe were compared with earlier reported biosensors, as shown in Tables 1 and 2 .

\subsection{Selectivity of $\mathbf{P b}^{2+}$ and Epn}

Selectivity is another important issue when assessing the performance of a newly proposed sensor, and the LFGr-ssDNAbased biosensor showed remarkably high selectivity toward $\mathrm{Pb}^{2+}$ and Epn (Fig. 11d and 12d). To investigate the selectivity of

Table 1 A comparison of different sensing probes used to determine $\mathrm{Pb}^{2+}$ ions $^{a}$

\begin{tabular}{lllll}
\hline Method & Sensing probe & LOD & Linear range & Real sample \\
\hline FM & T30695/SYBER green & $18 \mathrm{nM}$ & 0 to $480 \mathrm{nM}$ & Lake water \\
FM & AuNPs on GO & $10 \mathrm{nM}$ & 50 to $1000 \mathrm{nM}$ & Tap water \\
FM & NMSET/FAM-AuNPs & $10 \mathrm{nM}$ & 12.5 to $100 \mathrm{nM}$ & Tap water \\
FM & DNA-AgNCs & $3 \mathrm{nM}$ & 5 to $50 \mathrm{nM}$ & Tap and lake water \\
FM & Aptamer-functionalized UCAuNPs & $5.7 \mathrm{nM}$ & 25 to $1400 \mathrm{nM}$ & Tea and wastewater \\
FM & NMc-CuTA/LFGr-SsDNA/AO & $1.5 \mathrm{nM}$ & 0 to $100 \mathrm{nM}$ & Urine
\end{tabular}

${ }^{a}$ FM: fluorescence method; LOD: limit of detection; AuNPs: gold nanoparticles; GO: graphene oxide; NMSET: nanomaterial surface energy transfer; AgNCs: silver nanoclusters; UCAuNPs: upconversion gold nanoparticles. 
Table 2 A comparison of different sensing probes used to determine the drug Epn ${ }^{a}$

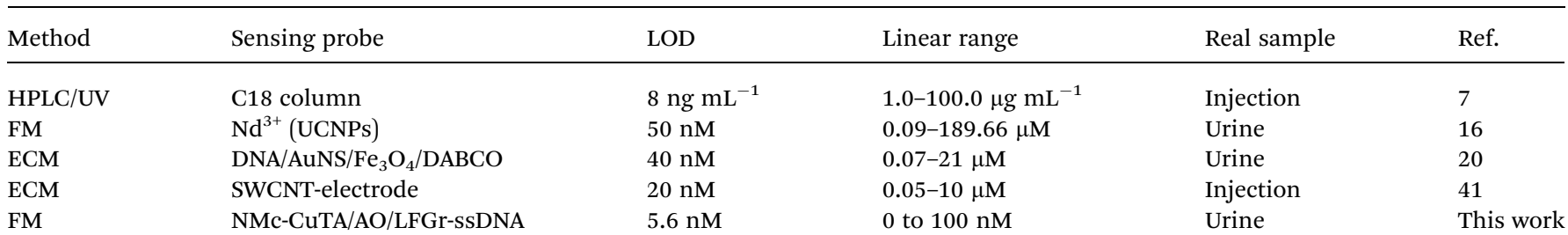

${ }^{a}$ FM: fluorescence method; LOD: limit of detection; ECM: electrochemical method; AuNS: gold nanostructure; DABCO: diazoniabicyclo[2.2.2] octane; SWCNT: single-walled carbon nanotube; UCNPs: upconversion nanoparticles.

Table 3 The determination of $\mathrm{Pb}^{2+}$ ion and Epn drug concentrations in RCS using the proposed sensor

\begin{tabular}{|c|c|c|c|}
\hline Sample & Spiked (nM) & Detected $\pm \mathrm{SD}(\mathrm{nM})$ & $\begin{array}{l}\text { Recovery } \\
(\%)\end{array}$ \\
\hline \multicolumn{4}{|l|}{$\mathbf{P b}^{2+}$} \\
\hline \multirow[t]{3}{*}{ Urine-1 } & 2 & $1.953^{x} \pm 0.43^{y}$ & 97.65 \\
\hline & 4 & $3.955^{x} \pm 0.51^{y}$ & 98.87 \\
\hline & 6 & $5.956^{x} \pm 1.29^{y}$ & 99.26 \\
\hline
\end{tabular}

Epn

$\begin{array}{llll}\text { Urine-2 } & 6 & 5.802^{x} \pm 0.63^{y} & 96.70 \\ & 8 & 7.752^{x} \pm 0.56^{y} & 96.90 \\ & 10 & 9.721^{x} \pm 1.43^{y} & 97.21\end{array}$

$x=$ Mean values of three determinations, $y=$ standard deviation.

the NMc-CuTA/LFGr-ssDNA/AO complex, its responses to various metal ions were recorded, as shown in Fig. 11d. From the results, it is clearly shown that $500 \mathrm{nM} \mathrm{Pb}^{2+}$ could induce a significant fluorescence enhancement in the sensing system but all other metal ions $\left(\mathrm{Hg}^{2+}, \mathrm{Cu}^{2+}, \mathrm{Ag}^{+}, \mathrm{Ni}^{2+}, \mathrm{Zn}^{2+}, \mathrm{Mg}^{2+}, \mathrm{K}^{+}\right.$, $\mathrm{Cr}^{3+}, \mathrm{Mn}^{2+}$, etc.) at concentrations of $1000 \mathrm{nM}$ did not result in an obvious fluorescence enhancement. It should be noted that $\mathrm{Pb}^{2+}$ ions form stable $\mathrm{G} 4$ complexes, as there are shorter $\mathrm{M}-\mathrm{O}$ and $\mathrm{O}-\mathrm{O}$ bonds than those stabilized by $\mathrm{K}^{+}{ }^{34}$ The high selectivity of the developed $\mathrm{Pb}^{2+}$ sensor may result in different $\mathrm{Pb}^{2+}$ stabilized G4 complex structures compared to $\mathrm{K}^{+}$-stabilized G4 complex structures. The selectivity of the Epn sensor was evaluated using the NMc-CuTA/LFGr-ssDNA/AO system after $\mathrm{Pb}^{2+}$ was successfully eliminated via the introduction of Cys. The selectivity of the Epn sensor system was investigated using other common drug biomolecules, such as IMT, AMP, STR, and TMF. The fluorescence intensity was not enhanced upon the addition of other drug biomolecules but it was significantly enhanced during the addition of Epn due to the formation of hydrogen and covalent bonds between Epn and DNA guanine base pairs. These interactions can be stabilized via covalent-bondmediated cellular formaldehyde, resulting in the formation of an Epn-G4 complex. ${ }^{35}$ These results indicate that the system possesses excellent selectivity for epirubicin and no interference from other drug biomolecules was seen (Fig. 12d).

\subsection{Analysis of $\mathbf{P b}^{2+}$ and Epn in $\mathrm{RS}$}

The practical applicability of this (NMc-CuTA/LFGr-ssDNA/AO) sensing strategy was evaluated through the detection of $\mathrm{Pb}^{2+}$ and Epn in real samples. Real samples, i.e., urine, were obtained from human subjects, and their consent was recorded through SRM Hospital, Tamil Nadu, India. Before the fluorescence sensing analysis of $\mathrm{Pb}^{2+}$ and Epn, all urine samples were pretreated. $50 \mathrm{~mL}$ of sample was filtered through a $0.2 \mu \mathrm{m}$ Millipore membrane and ultrasonicated for $20 \mathrm{~min}$. Then the filtrate was centrifuged for $20 \mathrm{~min}$ at $400 \mathrm{rpm}$. The supernatant was collected for fluorescence analysis. Then, the supernatant urine samples were spiked with standard $\mathrm{Pb}^{2+}$ and Epn solutions. Every sample was measured three times, and the results are listed in Table 3. Satisfactory recoveries of $\mathrm{Pb}^{2+}$ and Epn were obtained in the ranges of 97.65 to $99.26 \%$ and 96.70 to $97.21 \%$, respectively. This shows that our fluorescent sensor could accurately detect $\mathrm{Pb}^{2+}$ and Epn concentrations in urine samples.

\section{Conclusions}

In summary, we have reported a novel Turn-ON fluorescent biosensor for the sequential detection of $\mathrm{Pb}^{2+}$ and the cancer drug Epn based on self-assembled NMc-CuTA/LFGr-ssDNA/AO. The proposed sensor utilizes the remarkable fluorescence quenching abilities of NMc-CuTA via interactions with an LFGrsSDNA/AO probe, which has been extensively studied as a versatile sensing platform. The system works via a mechanism involving intermolecular structural changes in LFGr-ssDNA upon the introduction of $\mathrm{Pb}^{2+}$ and Epn, leading to the recovery of the fluorescence of $\mathrm{AO}$. The changes in fluorescence intensity with specificity as a result of SsDNA were used for the selective detection of $\mathrm{Pb}^{2+}$ ions and Epn with detection limits of 1.5 and $5.6 \mathrm{nM}$, respectively, which are superior to the values for earlier reported sensing systems. Therefore, this sensing system holds great potential for the practical testing of complicated samples (like human urine) with satisfying results. We anticipate that this report of a novel fluorescent biosensor could prompt the development of more sensitive, simple, highly selective, and label-free methods for $\mathrm{Pb}^{2+}$ and Epn detection by the scientific community. Moreover, this sensor strategy can be extended to detect other heavy metal ions and other biomolecules via modifications to the specific oligonucleotide base sequence.

\section{Conflicts of interest}

The authors declare no competing financial interests. 


\section{Acknowledgements}

The authors acknowledge financial support from the Department of Chemistry, SRM Institute of Science and Technology, Tamil Nadu-603 203, India.

\section{References}

1 R. Gorkhali, K. Huang, M. Kirberger and J. J. Yang, Metallomics, 2016, 8, 563-578.

2 H. Jin, D. Zhang, Y. Liu and M. Wei, RSC Adv. , 2020, 10, 66476653.

3 P. Rajaji and P. Panneerselvam, ACS Omega, 2020, 5, 2518825198.

4 A. Shams and A. Yari, Sens. Actuators, B, 2019, 286, 131-138. 5 S. Duan, W. K. Bleibel, R. S. Huang, S. J. Shukla, X. Wu, J. A. Badner and M. E. Dolan, Cancer Res., 2007, 67, 54255433.

6 C. R. T. Tarley, F. N. Andrade, F. M. de Oliveira, M. Z. Corazza, L. F. M. de Azevedo and M. G. Segatelli, Anal. Chim. Acta, 2011, 703, 145-151.

7 M. Tariq, S. Thomas, A. Singh and S. Talegaonkar, Braz. J. Pharm. Sci., 2018, 54(4), e17515.

8 G. Yang, C. Zhang, Q. Hu and J. Yin, J. Chromatogr. Sci., 2003, 41, 195-199.

9 S. A. R. Alavi-Tabari, M. A. Khalilzadeh, H. Karimi-Maleh and D. Zareyee, New J. Chem., 2018, 42, 3828-3832.

10 S. Yang, P. Liu, Y. Wang, Z. Guo, R. Tan and L. Qu, RSC Adv., 2020, 10, 18526-18532.

11 R. Gao, Z. Hu, X. Chang, Q. He, L. Zhang, Z. Tu and J. Shi, J. Hazard. Mater., 2009, 172, 324-329.

12 Y. Wei, B. Li, X. Wang and Y. Duan, Analyst, 2014, 139, 16181621.

13 M. Muppidathi, P. Perumal, R. Ayyanu and S. Subramanian, Analyst, 2019, 144, 3111-3118.

14 G. Liu, L. Zhang, D. Dong, Y. Liu and J. Li, Anal. Methods, 2016, 8, 7040-7046.

15 Y. Wang, M. Lv, Z. Chen, Z. Deng, N. Liu, J. Fan and W. Zhang, Front. Chem., 2020, 8, 1-8.

16 J. Mo, L. Shen, Q. Xu, J. Zeng, J. Sha, T. Hu, K. Bi and Y. Chen, Nanomaterials, 2019, 9(12), 1700.

17 X. Li, G. Wang, X. Ding, Y. Chen, Y. Gou and Y. Lu, Phys. Chem. Chem. Phys., 2013, 15, 12800-12804.

18 A. Khodadadi, E. Faghih-Mirzaei, H. Karimi-Maleh, A. Abbaspourrad, S. Agarwal and V. K. Gupta, Sens. Actuators, B, 2019, 284, 568-574.
19 S. Krishnan, S. Chatterjee, A. Solanki, N. Guha, M. K. Singh, A. K. Gupta and D. K. Rai, ACS Appl. Nano Mater., 2020, 3(11), 11203-11216.

20 A. B. Hashkavayi and J. B. Raoof, Biosens. Bioelectron., 2017, 91, 650-657.

21 G. Wang, J. Qin, X. Zhou, Y. Deng, H. Wang, Y. Zhao and J. Wei, Adv. Funct. Mater., 2018, 28, 1-8.

22 J. Wei, G. Wang, F. Chen, M. Bai, Y. Liang, H. Wang, D. Zhao and Y. Zhao, Angew. Chem., Int. Ed., 2018, 57, 9838-9843.

23 B. Liu and J. Liu, ACS Appl. Mater. Interfaces, 2015, 7, 2483324838.

24 C. Lu, Z. Huang, B. Liu, Y. Liu, Y. Ying and J. Liu, Angew. Chem., Int. Ed., 2017, 56, 6208-6212.

25 M. A. Rahim, H. Ejima, K. L. Cho, K. Kempe, M. Müllner, J. P. Best and F. Caruso, Chem. Mater., 2014, 26, 1645-1653. 26 Z. Fu and R. Chen, J. Anal. Methods Chem., 1980, 35, 43-49. 27 H. Huang, J. Qin, G. Wang, Z. Guo, X. Yu, Y. Zhao and J. Wei, CrystEngComm, 2018, 20, 7626-7630.

28 K. Rajar, R. A. Soomro, Z. H. Ibupoto, S. Uddin and A. Balouch, Int. J. Food Prop., 2017, 20, 1359-1367.

29 K. Ganesan, V. K. Jothi, A. Natarajan, A. Rajaram, S. Ravichandran and S. Ramalingam, Arabian J. Chem., 2020, 13, 6802-6814.

30 J. R. Günter, J. Solid State Chem., 1980, 35, 43-49.

31 D. S. Kozak, R. A. Sergiienko, E. Shibata, A. Iizuka and T. Nakamura, Sci. Rep., 2016, 6, 1-9.

32 H. Wu, H. Sun, W. Hong, L. Mao and Y. Liu, ACS Appl. Mater. Interfaces, 2017, 9, 32255-32263.

33 T. Li, S. Dong and E. Wang, J. Am. Chem. Soc., 2010, 132, 13156-13157.

34 F. W. Kotch, J. C. Fettinger and J. T. Davis, Org. Lett., 2000, 2, 3277-3280.

35 F. Yang, S. S. Teves, C. J. Kemp and S. Henikoff, Biochim. Biophys. Acta, Rev. Cancer, 2014, 1845, 84-89.

36 S. Zhan, Y. Wu, Y. Luo, L. Liu, L. He, H. Xing and P. Zhou, Anal. Biochem., 2014, 462, 19-25.

37 X. Fu, T. Lou, Z. Chen, M. Lin, W. Feng and L. Chen, ACS Appl. Mater. Interfaces, 2012, 4, 1080-1086.

38 C. Liu and C. Z. Huang, Fenxi Huaxue, 2014, 42, 1195-1198.

39 B. Zhang and C. Wei, Talanta, 2018, 182, 125-130.

40 M. Chen, M. Hassan, H. Li and Q. Chen, Microchim. Acta, 2020, 187(1), 85.

41 H. Zhang, J. Nanopart. Res., 2004, 6, 665-669. 